\title{
Roles of preoperative C-reactive protein are more relevant in buccal cancer than other subsites
}

\author{
Shiao Fwu Tai ${ }^{1,2}$, Huei-Tzu Chien ${ }^{3}$, Chi-Kuang Young ${ }^{4}$, Chung-Kang Tsao ${ }^{5}$, Alba de Pablo ${ }^{5}$, Kang-Hsing Fan ${ }^{6}$, \\ Chun-Ta Liao ${ }^{1,2}$, Hung-Ming Wang ${ }^{7}$, Chung-Jan Kang ${ }^{1,2}$, Joseph Tung-Chieh Chang ${ }^{6}$ and Shiang-Fu Huang ${ }^{1,3^{*}}$
}

\begin{abstract}
Background: C-reactive protein (CRP) is an early marker for inflammation, and a relationship between serum CRP levels and survival in oral cancer has been demonstrated previously. In this study, we investigated the roles of CRP in different oral cancer subsites.

Methods: Three hundred and forty-three oral squamous cell carcinoma patients between June 1999 and March 2015 were retrospectively reviewed. Serum CRP levels were measured preoperatively.

Results: The elevation of CRP levels ( $\geq 5.0 \mathrm{mg} / \mathrm{L}$ ) was significantly correlated with pathologic tumor status, pathologic nodal status, nodal extracapsular spread, tumor stage, skin invasion, tumor depth ( $\geq 10 \mathrm{~mm})$, and bone invasion. The correlation between elevation of CRP and clinicopathologic factors was more evident in the buccal cancer compared to other tumor subsites. The disease-free survival and overall survival correlation was significant in buccal cancer ( $p=0.003$ and $p<0.001)$ but not in tongue cancer $(p=0.119$ and $p=0.341)$ or other oral cancer subsites $(p=0.246$ and $p=0.696)$.
\end{abstract}

Conclusions: Preoperative serum CRP level was a prognosticator in oral squamous cell carcinoma, and its effect was more prominent in buccal cancer that occurs more frequently in areca-quid (AQ) endemic regions.

Keywords: Oral squamous cell carcinoma, C-reactive protein, Prognosticator

\section{Background}

Oral cavity cancer is a malignancy with increased incidence in recent years. As it is widely known, alcohol, betel nut, and cigarette consumption increased the risks of oral cavity cancer $[1,2]$. Chronic exposure to these carcinogenic factors can cause transform the oral cavity mucosa into malignancy. Part of the Taiwanese population commonly consumes cigarettes and betel nuts; it makes the oral cancer fifth in the top ten common cancers in Taiwan, and its incidence still increases in recent years [3]. For oral cancer treatments, a decision of surgical intervention, radiotherapy, chemotherapy, or

\footnotetext{
* Correspondence: bigmac@adm.cgmh.org.tw

'Department of Otolaryngology, Chang Gung Memorial Hospital, Linkou, Taiwan

${ }^{3}$ Department of Public Health, Chang Gung University, Taoyuan, Taiwan,

Republic of China

Full list of author information is available at the end of the article
}

combination, depends on cancer staging, lymph node metastasis, pathologic factors, and distant metastasis.

In recent years, more and more research studies proved that in addition to preoperative cancer staging, the patients' preoperative condition plays an important role in predicting the prognosis of oral cavity cancer. Inflammation, which may contribute to the formation of oral cavity cancer or was resulted from the host reaction to the tumor progression (Fig. 1), was also found to correlate with patient's prognosis [4]. Some inflammatory markers such as interleukin-6, tumor necrotic factor, and C-reactive protein (CRP) were recently identified as prognostic markers in oral cavity cancer [4-7]. CRP is an acute phase protein, which is synthesized by the liver and released into the bloodstream within several hours after tissue injury, being able to reflect infection or an inflammatory status [8]. In many human cancers, CRP has a role as a prognostic predicting factor $[5,7,9-11]$. 

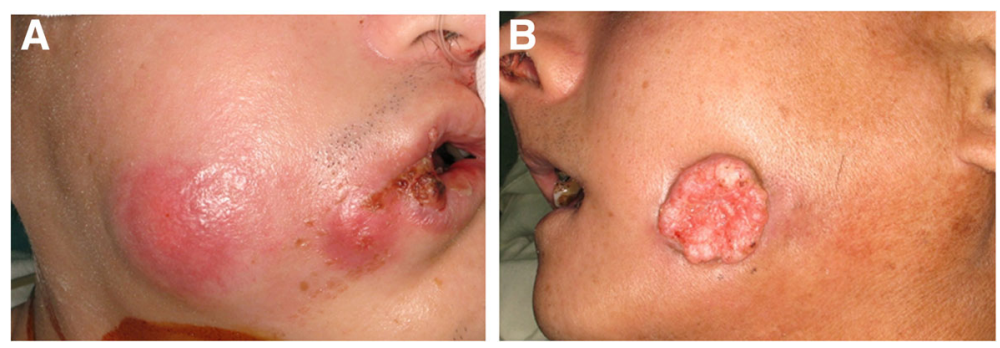

Fig. 1 Patients with same tumor invasion into the skin. a Combined with peri-tumoral inflammation. b Without peri-tumoral inflammation

We previously demonstrated that CRP was an independent prognostic factor in oral cavity squamous cell carcinoma (OSCC) [7]. However, the studies were limited by the case number and follow-up period. In this study, we expanded our patient number and included a longer follow-up period to clarify the prognostic role in OSCC. Also, we stratified our patients according to different tumor subsites. We hope that the analysis in this study will clarify the true prognostic value of CRP in OSCC.

\section{Methods \\ OSCC patients}

Three hundred and forty-three patients between June 1999 and March 2015 in Chang Gung Memorial Hospital were retrospectively recruited. The inclusion criteria were primary OSCC without previous treatment before. The exclusion criteria were patients with verrucous carcinoma or distant metastasis. All patients included in this study received radical surgery in curative intent and with or without adjuvant chemo-radiation therapy. After treatments, all the patients were followed up regularly in the clinic and ended on September 2015 or on the date that the patients expired.

\section{OSCC staging and treatment}

The patients in this series underwent an extensive preoperative survey, which included a detailed medical history and a complete physical examination, complete blood count, routine blood biochemistry, chest radiographs, computed tomography or magnetic resonance imaging scans of the head and neck, bone scan or positron emission tomography (PET), and liver ultrasound. The tumor staging followed the guidelines of the American Joint Committee on Cancer (7th edition) [12]. The tumor excisions in all patients were done by $\geq 1 \mathrm{~cm}$ safety margin. The tumor invasiveness parameters, which included tumor size, tumor cell differentiation, lymph node metastasis, lymph node extracapsular spread (ECS), depth of tumor invasion, perineural invasion, and soft tissue and lymphovascular invasion, were documented in the pathology report. For patients with advanced tumor stage (T3 or T4), lymph node ECS, tumor depth $\geq 10 \mathrm{~mm}$, or poor differentiation, postoperative radiotherapy or concomitant chemoradiotherapy would be suggested [13].

\section{Measurement of CRP}

The serum CRP level was measured preoperatively at the time of tissue diagnosis. It was measured before any medical treatment was delivered, including antibiotics $[6,13]$. The levels of CRPs were measured by an auto-analyzer (Hitachi Medico, Tokyo, Japan). Elevation of serum CRP level was defined at a cut point of $\geq 5.0 \mathrm{mg} / \mathrm{L}$.

\section{Statistical analysis}

The mean values of preoperative CRP in different tumor subistes were compared using ANOVA. Chi-square test with univariate analysis were used in this study. Survival differences were compared with the log-rank test. SPSS software, version 18.0 (SPSS, Chicago, IL, USA), was used for data analysis. A two-sided $p$ value $\leq 0.05$ was defined as statistically significant.

\section{Ethics approval and consent to participate}

All patients signed informed consent for participation of this study. This study had ethics approval and consent by the ethic committee in Chang Gung Memorial Hospital (Institutional Review Board of Chang Gung Medical Foundation, IRB No. 201600949B0), Taiwan, Republic of China on August 24, 2016.

\section{Results}

\section{Patient characteristics}

The clinical information of 343 OSCC patients in this study were shown in Table 1 (318 men and 25 women) and in Additional file 1 . Tongue $(n=132,38.5 \%)$ and buccal mucosa $(n=126,36.7 \%)$ were the most common OSCC sites in this study. Pathological tumor stage distributed as stage I $(n=76,22.2 \%)$, stage II $(n=66,19.2 \%)$, stage III $(n=43,12.5 \%)$, stage IVa $(n=133,38.8 \%)$, and stage IVb $(n=25,7.3 \%)$. The mean preoperative CRP was $6.96 \mathrm{mg} / \mathrm{L}$ (standard deviation (SD) \pm 12.06 ). The tumor stages were not different between different tumor subsites $(p=0.100)$. The mean CRP level was $5.90 \mathrm{mg} / \mathrm{L}( \pm \mathrm{SD}$ $10.53), 8.37 \mathrm{mg} / \mathrm{L}( \pm \mathrm{SD} 15.01)$, and $6.52 \mathrm{mg} / \mathrm{L}( \pm \mathrm{SD} 8.90)$ in tongue cancer, buccal cancer, and other oral cavity 
Table 1 Characteristics of 343 patients with OSCC

\begin{tabular}{|c|c|}
\hline Characteristic & Value \\
\hline \multicolumn{2}{|l|}{ Age (year) } \\
\hline Mean $( \pm$ SD) & $52.21( \pm 11.00)$ \\
\hline Range & $27.0-84.0$ \\
\hline \multicolumn{2}{|l|}{ Gender } \\
\hline Male & $25(7.3 \%)$ \\
\hline Female & $318(92.7 \%)$ \\
\hline \multicolumn{2}{|l|}{ Site of primary cancer } \\
\hline Tongue & $132(38.5 \%)$ \\
\hline Mouth floor & $12(3.5 \%)$ \\
\hline Lip & $24(7.0 \%)$ \\
\hline Buccal mucosa & $126(36.7 \%)$ \\
\hline Alveolar ridge & $31(9.0 \%)$ \\
\hline Hard palate & $2(0.6 \%)$ \\
\hline Retromolar trigone & $16(4.7 \%)$ \\
\hline \multicolumn{2}{|l|}{ Pathologic tumor status } \\
\hline 1 & $91(26.5 \%)$ \\
\hline 2 & $119(34.7 \%)$ \\
\hline 3 & $24(7.0 \%)$ \\
\hline $4 a$ & $85(24.8 \%)$ \\
\hline $4 b$ & $24(7.0 \%)$ \\
\hline \multicolumn{2}{|l|}{ Pathologic N stage } \\
\hline NO & $201(58.6 \%)$ \\
\hline N1 & $47(13.7 \%)$ \\
\hline $\mathrm{N} 2 \mathrm{~b}$ & $79(23.0 \%)$ \\
\hline $\mathrm{N} 2 \mathrm{C}$ & $15(4.4 \%)$ \\
\hline N3 & $1(0.3 \%)$ \\
\hline \multicolumn{2}{|l|}{ Pathologic stage } \\
\hline । & $76(22.2 \%)$ \\
\hline$\|$ & $66(19.2 \%)$ \\
\hline III & $43(12.5 \%)$ \\
\hline $\mathrm{IVa}$ & $133(38.8 \%)$ \\
\hline $\mathrm{IVb}$ & $25(7.3 \%)$ \\
\hline
\end{tabular}

cancers, respectively. The CRP levels were not significantly different between subsites (ANOVA $p=0.240$ ).

\section{Association between CRP level with clinicopathologic parameters and prognosis}

The relationship between CRP levels and tumor clinicopathologic parameters in OSCCs were examined and showed in Table 2. Advanced tumor status $(p<0.001)$, tumor stage $(p<0.001)$, skin invasion $(p<0.001)$, bone invasion $(p<0.001)$, and tumor depth $\geq 10 \mathrm{~mm}(p<0.001)$ and advanced pathologic nodal status $(p=0.006)$ and lymphatic invasion $(p=0.068)$ were significantly correlated with $C R P$ elevation $(C R P \geq 5.0 \mathrm{mg} / \mathrm{L})$.
Table 2 Association between CRP and clinicopathologic parameters $(n=343)$

\begin{tabular}{|c|c|c|c|c|c|}
\hline \multirow[t]{2}{*}{ Characteristic } & \multicolumn{4}{|l|}{ CRP } & \multirow[t]{2}{*}{$p$ value } \\
\hline & \multicolumn{2}{|c|}{ Negative } & \multicolumn{2}{|c|}{ Positive } & \\
\hline \multicolumn{6}{|l|}{ Pathologic tumor status } \\
\hline Early $(T 1-T 2)(n=210)$ & 181 & $(86.2 \%)$ & 29 & (13.8\%) & $<0.001$ \\
\hline Advanced (T3-T4) $(n=133)$ & 68 & $(51.1 \%)$ & 65 & $(48.9 \%)$ & \\
\hline \multicolumn{6}{|l|}{ Pathologic N stage } \\
\hline NO $(n=201)$ & 156 & $(77.6 \%)$ & 45 & (22.4\%) & 0.006 \\
\hline $\mathrm{N} 1(n=47)$ & 36 & $(76.6 \%)$ & 11 & $(23.4 \%)$ & $0.002^{\mathrm{a}}$ \\
\hline $\mathrm{N} 2(n=94)$ & 57 & $(60.6 \%)$ & 37 & (39.4\%) & \\
\hline N3 $(n=1)$ & 0 & $(0.0 \%)$ & 1 & $(100.0 \%)$ & \\
\hline \multicolumn{6}{|l|}{ Nodal status } \\
\hline$(-)$ metastasis, $(-)$ ECS $(n=201)$ & 156 & $(77.6 \%)$ & 45 & (22.4\%) & 0.006 \\
\hline$(+)$ metastasis, $(-)$ ECS $(n=59)$ & 44 & $(74.6 \%)$ & 15 & (25.4\%) & $0.002^{\mathrm{a}}$ \\
\hline$(+)$ metastasis, $(+)$ ECS $(n=83)$ & 49 & $(59.0 \%)$ & 34 & $(41.0 \%)$ & \\
\hline \multicolumn{6}{|l|}{ Differentiation } \\
\hline Well $(n=107)$ & 77 & $(72.0 \%)$ & 30 & $(28.0 \%)$ & 0.308 \\
\hline Moderate $(n=192)$ & 144 & $(75.0 \%)$ & 48 & (25.0\%) & $0.538^{\mathrm{a}}$ \\
\hline Poor $(n=44)$ & 28 & $(63.6 \%)$ & 16 & $(36.4 \%)$ & \\
\hline \multicolumn{6}{|l|}{ Tumor stage } \\
\hline Early (I-II) $(n=142)$ & 123 & $(86.6 \%)$ & 19 & (13.4\%) & $<0.001$ \\
\hline Advanced (III-IV) $(n=201)$ & 126 & $(62.7 \%)$ & 75 & $(37.3 \%)$ & \\
\hline \multicolumn{6}{|l|}{ Skin invasion } \\
\hline No $(n=304)$ & 231 & $(76.0 \%)$ & 73 & $(24.0 \%)$ & $<0.001$ \\
\hline Yes $(n=39)$ & 18 & $(46.2 \%)$ & 21 & $(53.8 \%)$ & \\
\hline \multicolumn{6}{|l|}{ Nerve invasion } \\
\hline No $(n=229)$ & 171 & $(74.7 \%)$ & 58 & (25.3\%) & 0.221 \\
\hline Yes $(n=114)$ & 78 & $(68.4 \%)$ & 36 & $(31.6 \%)$ & \\
\hline \multicolumn{6}{|l|}{ Blood vessel invasion } \\
\hline No $(n=331)$ & 242 & (73.1\%) & 89 & $(26.9 \%)$ & $0.322^{b}$ \\
\hline Yes $(n=12)$ & 7 & $(58.3 \%)$ & 5 & $(41.7 \%)$ & \\
\hline \multicolumn{6}{|l|}{ Lymphatic invasion } \\
\hline No $(n=334)$ & 245 & $(73.4 \%)$ & 89 & $(26.6 \%)$ & $0.068^{b}$ \\
\hline Yes $(n=9)$ & 4 & $(44.4 \%)$ & 5 & $(55.6 \%)$ & \\
\hline \multicolumn{6}{|l|}{ Tumor depth ( $\geq 10 \mathrm{~mm})$} \\
\hline No $(n=160)$ & 137 & $(85.6 \%)$ & 23 & $(14.4 \%)$ & $<0.001$ \\
\hline Yes $(n=183)$ & 112 & $(61.2 \%)$ & 71 & (38.8\%) & \\
\hline \multicolumn{6}{|l|}{ Bone invasion } \\
\hline No $(n=275)$ & 215 & $(78.2 \%)$ & 60 & (21.8\%) & $<0.001$ \\
\hline Yes $(n=68)$ & 34 & $(50.0 \%)$ & 34 & (50.0\%) & \\
\hline
\end{tabular}

ECS extracapsular spread

${ }^{a} X^{2}$ trend test

${ }^{\mathrm{b}}$ Fisher's exact test

We further analyzed the association between CRP and clinicopathologic factors in different tumor sites (buccal, tongue, and other locations). In buccal cancer (Table 3), 
Table 3 Association between CRP and clinicopathologic parameters in buccal cancer $(n=126)$

$\begin{array}{ll}\text { Characteristic } & \text { CRP } \\ & \text { Negative Positive }\end{array}$

Pathologic tumor status

Early (T1-T2) $(n=71)$

$60 \quad(84.5 \%) \quad 11 \quad(15.5 \%) \quad<0.001$

Advanced (T3-T4) ( $n=55)$

$25 \quad(45.5 \%) \quad 30 \quad(54.5 \%)$

Pathologic $\mathrm{N}$ stage

$\begin{array}{llllll}\text { N0 }(n=70) & 52 & (74.3 \%) & 18 & (25.7 \%) & 0.009 \\ \text { N1 }(n=18) & 15 & (83.3 \%) & 3 & (16.7 \%) & 0.006^{\mathrm{a}} \\ \text { N2 }(n=37) & 18 & (48.6 \%) & 19 & (51.4 \%) & \\ \text { N3 }(n=1) & 0 & (0.0 \%) & 1 & (100.0 \%) & \end{array}$

Nodal status

$\begin{array}{llllll}(-) \text { metastasis, }(-) \text { ECS }(n=70) & 52 & (74.3 \%) & 18 & (25.7 \%) & 0.003 \\ (+) \text { metastasis, }(-) \text { ECS }(n=27) & 21 & (77.8 \%) & 6 & (22.2 \%) & 0.005^{\text {a }}\end{array}$

$(+)$ metastasis, $(+)$ ECS $(n=29) \quad 12 \quad(41.4 \%) \quad 17 \quad(58.6 \%)$

Differentiation

$\begin{array}{llllll}\text { Well }(n=48) & 28 & (58.3 \%) & 20 & (41.7 \%) & 0.211 \\ \text { Moderate }(n=62) & 46 & (74.2 \%) & 16 & (25.8 \%) & 0.192^{\mathrm{a}} \\ \text { Poor }(n=16) & 11 & (68.8 \%) & 5 & (31.2 \%) & \end{array}$

Tumor stage

Early $(I-I)(n=48)$

$41 \quad(85.4 \%) \quad 7 \quad(14.6 \%) \quad 0.001$

Advanced (III-IV) $(n=78)$

Skin invasion

No $(n=96)$

Yes $(n=30)$

Nerve invasion

$$
\text { No }(n=86)
$$

Yes $(n=40)$

Blood vessel invasion

$$
\begin{aligned}
& \text { No }(n=122) \\
& \text { Yes }(n=4)
\end{aligned}
$$

Lymphatic invasion

$$
\begin{aligned}
& \text { No }(n=122) \\
& \text { Yes }(n=4)
\end{aligned}
$$

Tumor depth $\geq 10 \mathrm{~mm}$

$$
\begin{aligned}
& \text { No }(n=60) \\
& \text { Yes }(n=66)
\end{aligned}
$$$$
41 \quad(85.4 \%) \quad 7 \quad(14.6 \%)
$$$$
44 \quad(56.4 \%) \quad 34 \quad(43.6 \%)
$$

0.001

Bone invasion

\begin{tabular}{llllll} 
No $(n=92)$ & 68 & $(73.9 \%)$ & 24 & $(26.1 \%)$ & 0.011 \\
Yes $(n=34)$ & 17 & $(50.0 \%)$ & 17 & $(50.0 \%)$ & \\
\hline
\end{tabular}

ECS extracapsular spread

${ }^{a} x^{2}$ trend test

${ }^{\mathrm{b}}$ Fisher's exact test

CRP elevation $(C R P \geq 5.0 \mathrm{mg} / \mathrm{L})$ was significantly associated with advanced tumor status $(p<0.001)$, advanced stage $(p=0.001)$, advanced pathological nodal status $(p=0.009)$, nodal status with ECS $(p=0.003)$, tumor depth $\geq 10 \mathrm{~mm} \quad(p<0.001)$, skin invasion $(p<0.001)$, nerve invasion $(p=0.042)$, and bone invasion $(p=$ $0.011)$.

In tongue cancer (Table 4), CRP elevation (CRP $\geq$ $5.0 \mathrm{mg} / \mathrm{L}$ ) showed a strong relationship with advanced pathological tumor status $(p<0.001)$ and correlated with advanced pathologic nodal status $(p=0.027)$ and advanced tumor stage $(p=0.021)$.

In other tumor subsites (Table 5), the CRP elevation $(\mathrm{CRP} \geq 5.0 \mathrm{mg} / \mathrm{L})$ was significantly associated with advanced pathological tumor status $(p=0.001)$, tumor stage $(p=0.009)$, and bone invasion $(p<0.001)$. It was also correlated with tumor depth $\geq 10 \mathrm{~mm}(p=0.009)$.

\section{The association between CRP and survival}

Comparing the prognosis between the two by univariate analysis, the group with lower CRP $(C R P<5.0 \mathrm{mg} / \mathrm{L})$ has a longer disease-free survival (DFS) than the high CRP group $(C R P \geq 5.0 \mathrm{mg} / \mathrm{L})$, (log-rank test $p \leq 0.001$, Fig. 2a). Similarly, overall survival (OS) is longer in the low CRP level group $(\mathrm{CRP}<5.0 \mathrm{mg} / \mathrm{L})$ compared to the high CRP level group (CRP $\geq 5.0 \mathrm{mg} / \mathrm{L}$ ) (log-rank test $p \leq 0.001$, Fig. $2 \mathrm{~b}$ ). The hazard ratio (HR) for CRP including all subsites, DFS 1.902 (95\% confidence interval (CI) 1.302-2.778) and OS 2.235 (95\% CI 1.393-3.585). We analyzed the influence of CRP on survival according to different subsites: the HR for CRP in tongue cancer DFS 1.785 (95\% CI $0.848-3.757$ ); OS 1.535 (95\% CI 0.630-3.741); the HR for CRP in buccal cancer, DFS 2.293 (95\% CI 1.309-4.017); OS 3.610 (95\% CI 1.732 7.526); the HR for CRP in other cancer subsites, DFS 1.577 (95\% CI $0.721-3.449$ ), OS 1.252 (95\% CI $0.403-$ $3.885)$.

\section{Discussion}

Our previous studies showed a positive relationship between CRP level elevation and advanced oral cavity cancer stage [6]. Therefore, CRP has the potential to be a biomarker for oral cavity cancer and a predictor of prognosis before treatment. In this study, we recruited more cases (343 cases) to evaluate the connection between preoperative serum CRP level, oral cavity cancer stage, and prognosis. In the present study, oral cavity cancer had a greater prevalence between males, with mean age falling in the middle age period. This distribution was probably due to greater exposure to oral cavity cancer risk factors (smoking, drinking, and chewing betel nut habit) [14-17] in this subgroup. In contrary, the most common site of oral cavity cancer in the Western population is the tongue. However, in Taiwan, due to betel nut chewing, the common sites of oral cavity cancer are buccal mucosa and tongue, compatible with our patients' tumor site distribution [18, 19]. 
Table 4 Association between CRP and clinicopathologic parameters in tongue cancer $(n=132)$

$\begin{array}{ll}\text { Characteristic CRP } & \\ & \text { Negative Palue }\end{array}$

Pathologic tumor status

$\begin{array}{llllll}\text { Early }(\mathrm{T} 1-\mathrm{T} 2)(n=96) & 83 & (86.5 \%) & 13 & (13.5 \%) & <0.001 \\ \text { Advanced (T3-T4) }(n=36) & 19 & (52.8 \%) & 17 & (47.2 \%) & \end{array}$

Pathologic N stage

$\begin{array}{llllll}\text { N0 }(n=77) & 64 & (83.1 \%) & 13 & (16.9 \%) & 0.027 \\ \text { N1 }(n=15) & 13 & (86.7 \%) & 2 & (13.3 \%) & 0.017^{\mathrm{a}} \\ \text { N2 }(n=40) & 25 & (62.5 \%) & 15 & (37.5 \%) & \end{array}$

Nodal status

$$
\begin{array}{llllll}
(-) \text { metastasis, }(-) \text { ECS }(n=77) & 64 & (83.1 \%) & 13 & (16.9 \%) & 0.163 \\
(+) \text { metastasis, }(-) \text { ECS }(n=17) & 12 & (70.6 \%) & 5 & (29.4 \%) & 0.066^{\text {a }} \\
(+) \text { metastasis, (+) ECS }(n=38) & 26 & (68.4 \%) & 12 & (31.6 \%) &
\end{array}
$$

Differentiation

$\begin{array}{llllll}\text { Well }(n=33) & 28 & (84.8 \%) & 5 & (15.2 \%) & 0.152 \\ \quad \text { Moderate }(n=81) & 63 & (77.8 \%) & 18 & (22.2 \%) & \\ \quad \text { Poor }(n=18) & 11 & (61.1 \%) & 7 & (38.9 \%) & \\ \text { Tumor stage } & & & & & \\ \quad \text { Early }(I-I I)(n=64) & 55 & (85.9 \%) & 9 & (14.1 \%) & 0.021 \\ \text { Advanced (III-IV) }(n=68) & 47 & (69.1 \%) & 21 & (30.9 \%) & \end{array}$

Skin invasion

$$
\begin{array}{llllll}
\text { No }(n=131) & 101 & (77.1 \%) & 30 & (22.9 \%) & 1.000 \\
\text { Yes }(n=1) & 1 & (100.0 \%) & 0 & (0.0 \%) &
\end{array}
$$

Nerve invasion

$$
\begin{array}{llllll}
\text { No }(n=77) & 60 & (77.9 \%) & 17 & (22.1 \%) & 0.833 \\
\text { Yes }(n=55) & 42 & (76.4 \%) & 13 & (23.6 \%) &
\end{array}
$$

Blood vessel invasion

$$
\begin{array}{llllll}
\text { No }(n=130) & 100 & (76.9 \%) & 30 & (23.1 \%) & 1.000^{\mathrm{b}} \\
\text { Yes }(n=2) & 2 & (100.0 \%) & 0 & (0.0 \%) &
\end{array}
$$

Lymphatic invasion

$$
\begin{array}{llllll}
\text { No }(n=129) & 101 & (78.3 \%) & 28 & (21.7 \%) & 0.129^{\mathrm{b}} \\
\text { Yes }(n=3) & 1 & (33.3 \%) & 2 & (66.7 \%) &
\end{array}
$$

Tumor depth $(\geq 10 \mathrm{~mm})$

$$
\begin{array}{llllll}
\text { No }(n=58) & 49 & (84.5 \%) & 9 & (15.5 \%) & 0.080 \\
\text { Yes }(n=74) & 53 & (71.6 \%) & 21 & (28.4 \%) &
\end{array}
$$

Bone invasion

$\begin{array}{llllll}\text { No }(n=129) & 100 & (77.5 \%) & 29 & (22.5 \%) & 0.542^{\mathrm{b}} \\ \text { Yes }(n=3) & 2 & (66.7 \%) & 1 & (33.3 \%) & \end{array}$

ECS extracapsular spread

${ }^{a} X^{2}$ trend test

${ }^{\mathrm{b}}$ Fisher's exact test

It is still a debate if the elevation of CRP could be due to a concomitant pulmonary infection or other infection, and be non-specific for oral cancer. In 18 of our cases, the CRP levels were checked twice. The first test was at
Table 5 Association between CRP and clinicopathologic parameters in subsites other than tongue and buccal mucosa $(n=85)$

\begin{tabular}{lllllll}
\hline Characteristic & \multicolumn{3}{l}{ CRP } & \multirow{2}{*}{$p$ value } \\
\cline { 2 - 5 } & Negative & \multicolumn{2}{l}{ Positive } & \\
\hline Pathologic tumor status & 38 & $(88.4 \%)$ & 5 & $(11.6 \%)$ & 0.001 \\
$\quad$ Early (T1-T2) $(n=43)$ & 24 & $(57.1 \%)$ & 18 & $(42.9 \%)$ & \\
Advanced (T3-T4) $(n=42)$ & & & & & \\
Pathologic N stage & 40 & $(74.1 \%)$ & 14 & $(25.9 \%)$ & 0.277 \\
N0 ( $n=54)$ & 8 & $(57.1 \%)$ & 6 & $(42.9 \%)$ & $0.765^{\text {a }}$ \\
N1 $(n=14)$ & 14 & $(82.4 \%)$ & 3 & $(17.6 \%)$ & \\
N2 $(n=17)$ & & & & &
\end{tabular}

Nodal status

$$
\begin{array}{llllll}
(-) \text { metastasis, }(-) \text { ECS }(n=54) & 40 & (74.1 \%) & 14 & (25.9 \%) & 0.915 \\
(+) \text { metastasis, }(-) \text { ECS }(n=15) & 11 & (73.3 \%) & 4 & (26.7 \%) & 0.694^{\text {a }} \\
(+) \text { metastasis, (+) ECS }(n=16) & 11 & (68.8 \%) & 5 & (31.2 \%) &
\end{array}
$$

Differentiation

$$
\begin{array}{llllll}
\text { Well }(n=26) & 21 & (80.8 \%) & 5 & (19.2 \%) & 0.425 \\
\quad \text { Moderate }(n=49) & 35 & (71.4 \%) & 14 & (28.6 \%) & 0.195^{\mathrm{a}} \\
\quad \text { Poor }(n=10) & 6 & (60.0 \%) & 4 & (40.0 \%) & \\
\text { Tumor stage } & & & & & \\
\quad \text { Early }(I-I I)(n=30) & 27 & (90.0 \%) & 3 & (10.0 \%) & 0.009 \\
\text { Advanced (III-IV) }(n=55) & 35 & (63.6 \%) & 20 & (36.4 \%) &
\end{array}
$$

Skin invasion

$$
\begin{array}{llllll}
\text { No }(n=77) & 57 & (74.0 \%) & 20 & (26.0 \%) & 0.677^{\text {a }} \\
\text { Yes }(n=8) & 5 & (62.5 \%) & 3 & (37.5 \%) &
\end{array}
$$

Nerve invasion

$\begin{array}{llllll}\text { No }(n=66) & 48 & (72.7 \%) & 18 & (27.3 \%) & 0.934 \\ \text { Yes }(n=19) & 14 & (73.7 \%) & 5 & (26.3 \%) & \end{array}$

Blood vessel invasion

$\begin{array}{llllll}\text { No }(n=79) & 59 & (74.7 \%) & 20 & (25.3 \%) & 0.337^{\mathrm{a}} \\ \text { Yes }(n=6) & 3 & (50.0 \%) & 3 & (50.0 \%) & \end{array}$

Lymphatic invasion

$$
\begin{array}{llllll}
\text { No }(n=83) & 60 & (72.3 \%) & 23 & (27.7 \%) & 0.383 \\
\text { Yes }(n=2) & 2 & (100.0 \%) & 0 & (0.0 \%) &
\end{array}
$$

Tumor depth $\geq 10 \mathrm{~mm}$

$$
\begin{array}{llllll}
\text { No }(n=42) & 36 & (85.7 \%) & 6 & (14.3 \%) & 0.009 \\
\text { Yes }(n=43) & 26 & (60.5 \%) & 17 & (39.5 \%) &
\end{array}
$$

Bone invasion

\begin{tabular}{llllll} 
No $(n=54)$ & 47 & $(87.0 \%)$ & 7 & $(13.0 \%)$ & $<0.001$ \\
Yes $(n=31)$ & 15 & $(48.4 \%)$ & 16 & $(51.6 \%)$ & \\
\hline
\end{tabular}

ECS extracapsular spread

${ }^{a} x^{2}$ trend test

† Fisher's exact test

the time of diagnosis, and the second test was performed the day before surgery. Eighty-three percent of the patients had similar levels or a more elevated CRP level in 

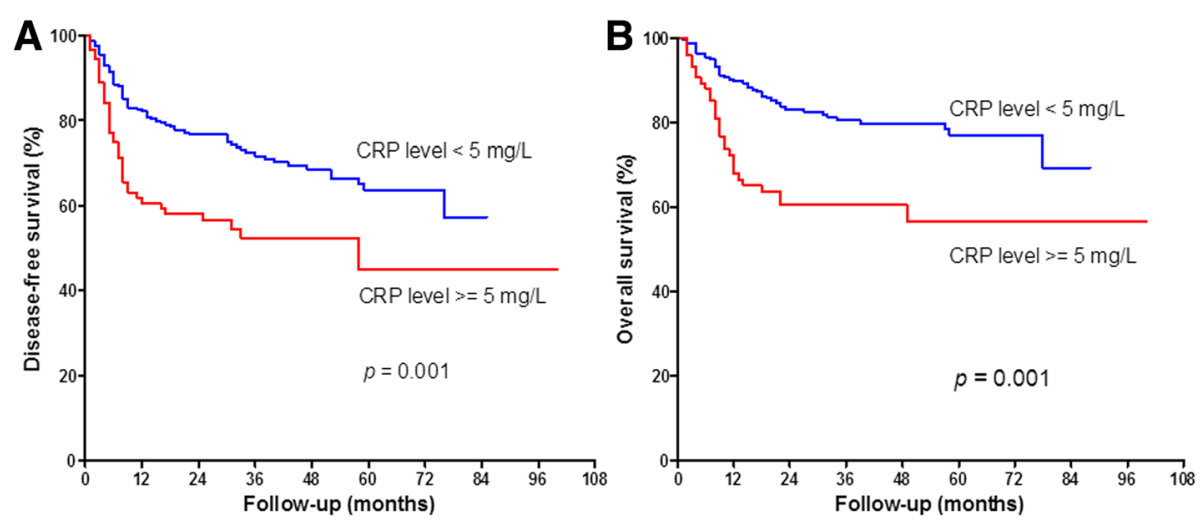

Fig. 2 Survival curves in 343 OSCC patients related to the preoperative CRP level. a The lower CRP level group showed significantly better DFS compared to the higher CRP group $(p<0.001)$. b The lower CRP level group showed significantly better OS compared to the higher CRP level group $(p<0.001)$

the second test; only three cases had lower CRP levels in the second test (Fig. 3, paired $t$ test $p=0.201$ ). This indicated that the serum CRP levels are stable in OSCC cancer patients, and so, the serum level analyzed in our study was not amenable to change in a different time period.

CRP is an acute phase protein as the host reacts to an inflammatory response and released from the liver into the bloodstream. Because of its short plasma half-life and robust reaction, it has been used clinically as a marker for inflammatory or infectious status [10,11,20,21]. Recently, CRP has proved a predictive factor in certain human cancers such as gastrointestinal, breast, lung, and gynecologic cancers $[9,22-26]$. There are three mechanisms about the relationship between CRP elevation and cancer prognosis: (1) oxidative damage caused by inflammation promotes tumor growth, (2) the tumor growth and apoptosis induced the release of CRP, (3) inflammation is a contributing factor to tumor progression and reflects in

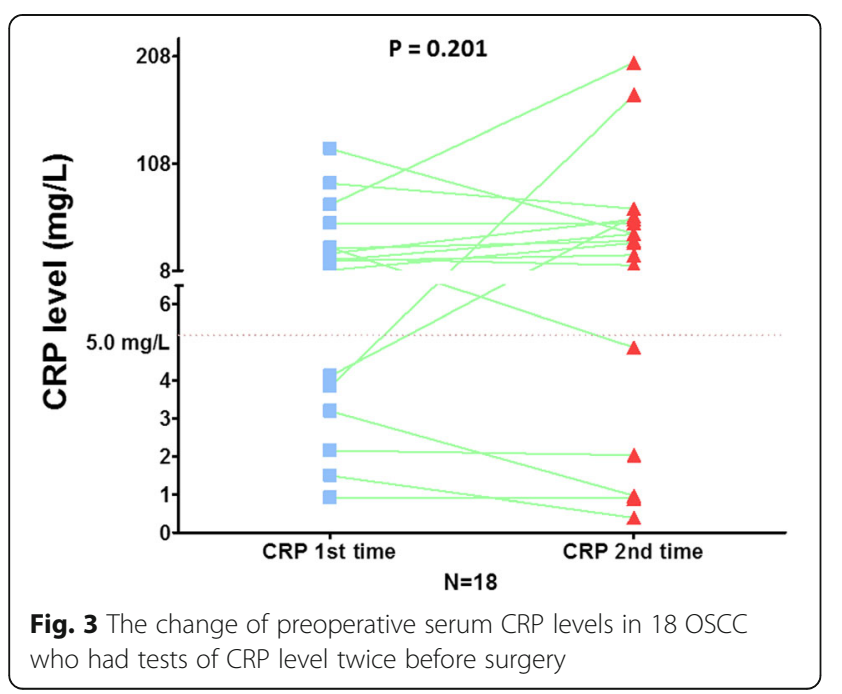

the elevation of CRP. We demonstrated that the CRP level was closely related with an increased squamous cell carcinoma antigen (SCC-Ag) and neutrophil to lymphocyte ratio (NLR) in peripheral blood tests at the time of diagnosis in OSCC patients [7, 27]. The SCCAg was closely related with primary tumor status and lymph node metastasis, which could stand for the tumor burden $[7,28,29]$. The elevated neutrophil ratio could be from tumor growth and the consequent immune response from the host. Circulating neutrophils contain and secrete various cytokines including circulating matrix metalloproteinases [30], vascular endothelial growth factor (VEGF) [31], platelet-derived growth factor, fibroblast growth factor, CXCL8 [32], elastases [33], and interleukin-8 [34]. These cytokines create a microenvironment that facilitates extracellular matrix remodeling, endothelial cell migration, and tumor cell invasion. Also, the released cytokines such as IL-6 could further stimulate the production of CRP in the liver [8].

In this study, CRP elevation and tumor status presented a clear positive correlation. To clarify the role of preoperative serum CRP level in OSCC, we stratified our patients into different subsites. There was a similar relationship between CRP elevation and tumor status for each cancer subsite. In the literature, nine studies investigated the roles of CRP in OSCC (Table 6) [6, 35-40]. Only one study did not find any association between CRP elevation and survival. Another point to consider is the fact that the included studies used inconsistent cut-off values for CRP levels, which caused heterogeneity in the influence of CPR in prognosis. In a meta-analysis in urologic malignancies, a similar condition of different cut-off points was observed, and they found setting the level at $5.0 \mathrm{mg} / \mathrm{L}$ was the most appropriate [41].

In this study, we found an elevated CRP was highly correlated with primary tumor status, tumor depth, and 
Table 6 Literature review for the relationship of CRP level and survival in OSCC patients

\begin{tabular}{|c|c|c|c|c|c|}
\hline Authors/year & Tumor sites/nations & Case no. & Cut-off point, tumor stage & Disease-free survival (DFS) & Overall survival (OS) \\
\hline $\begin{array}{l}\text { Jablonska E. et al. } \\
\text { (1997) [5] }\end{array}$ & OSCC/Poland & 42 & $\begin{array}{l}\text { Compares mean values, significant } \\
\text { difference between stages IV vs II } \\
(p<0.01)\end{array}$ & NA & NA \\
\hline $\begin{array}{l}\text { Khandavilli S.D. } \\
\text { et al. (2009) [36] }\end{array}$ & $\begin{array}{l}\text { OSCC/United } \\
\text { Kingdom }\end{array}$ & 60 & $\begin{array}{l}C R P>5.0 \mathrm{mg} / \mathrm{L} ; \text { significant with } \\
\text { tumor stage, } p=0.046\end{array}$ & NA & $\begin{array}{l}\text { Significant in OS } \\
(p<0.001) \text { (multivariate) }\end{array}$ \\
\hline $\begin{array}{l}\text { Kruse A.L. et al. } \\
\text { (2010) [37] }\end{array}$ & OSCC/Switzerland & 278 & $C R P>5.0 \mathrm{mg} / \mathrm{L} ; \mathrm{NA}$ & $\begin{array}{l}\text { No significant difference } \\
(p=0.137)\end{array}$ & $\begin{array}{l}\text { Not significant in time } \\
\text { to metastasis }(p=0.468)\end{array}$ \\
\hline $\begin{array}{l}\text { Chen, H.H. et al. } \\
\text { (2011) [6] }\end{array}$ & OSCC/Taiwan & 59 & $\begin{array}{l}C R P \geq 5.0 \mathrm{mg} / \mathrm{L}, \text { significant with } \\
\text { tumor stage, } p=0.005\end{array}$ & $\begin{array}{l}\text { Significant difference in 2-year } \\
\text { DFS, } p<0.001\end{array}$ & $\begin{array}{l}\text { Significant difference in } \\
\text { 2-year OS, } p=0.013\end{array}$ \\
\hline $\begin{array}{l}\text { Grimm, M. et al. } \\
\text { (2012) [45] }\end{array}$ & OSCC/Germany & 187 & $\begin{array}{l}C R P \geq 1.1 \mathrm{mg} / \mathrm{L} ; \mathrm{LPI} \text { significantly } \\
\text { correlated with advanced tumor } \\
\text { stage, } p=0.0002^{\mathrm{a}}\end{array}$ & 5-year DFS, $p<0.0001$ & NA \\
\hline $\begin{array}{l}\text { Huang SF et al. } \\
\text { (2012) [7] }\end{array}$ & OSCC/Taiwan & 142 & $\begin{array}{l}C R P \geq 5.0 \mathrm{mg} / \mathrm{L} \text {, significantly } \\
\text { related with tumor stage, } p=0.001\end{array}$ & 3-year DFS, $p<0.001$ & 3 -year OS, $p=0.008$ \\
\hline $\begin{array}{l}\text { Peter F. et al. } \\
\text { (2013) [38] }\end{array}$ & HNSCC/Germany & 261 & $\begin{array}{l}C R P \geq 2.0 \mathrm{mg} / \mathrm{L}, \text { significant with } \\
\text { advanced tumor stage, } p=0.006 \text {; } \\
\text { nodal status, } p=0.045\end{array}$ & NA & 5 -year OS, $p<0.0001$ \\
\hline $\begin{array}{l}\text { Chen, I.H. et al. } \\
\text { (2014) [39] }\end{array}$ & OSCC/Taiwan & $\begin{array}{l}\text { Recurrent } \\
\text { OSCC, } 100\end{array}$ & $\begin{array}{l}C R P \geq 5.0 \mathrm{mg} / \mathrm{L} \text {, significant with } \\
\text { tumor stage, } p<0.001\end{array}$ & NA & $\begin{array}{l}\text { Mean } 33.53 \text { months, OS } \\
\text { significantly different, } \\
p<0.001\end{array}$ \\
\hline $\begin{array}{l}\text { Farhan-Alanie, O.M. } \\
\text { et al. (2015) [40] }\end{array}$ & $\begin{array}{l}\text { OSCC and soft palatal } \\
\text { ca/ United Kingdom }\end{array}$ & 178 & $\begin{array}{l}\text { Modified Glasgow scale: combined } \\
\text { albumin }(<35 \mathrm{~g} / \mathrm{L}) \text { and CRP } \\
(>10 \mathrm{mg} / \mathrm{L}) \text { level }\end{array}$ & $\begin{array}{l}\text { Modified Glasgow scale related } \\
\text { with cancer-specific survival } \\
(p<0.001)\end{array}$ & $\begin{array}{l}\text { Modified Glasgow scale } \\
\text { related with overall } \\
\text { survival }(p<0.001)\end{array}$ \\
\hline
\end{tabular}

${ }^{\mathrm{a}}$ Combines $\mathrm{CRP}, \mathrm{Hb}$, and WBC as laboratory prognostic index (LPI)

lymph node metastasis in OSCCs. Regarding the tumor subsites, the elevation of CRP in buccal cancer had the most promising association with most clinicopathologic factors. In addition, the elevation of CRP was related with lymph node metastasis in buccal and tongue cancers, while the association between increased CRP and ECS was only found in buccal cancer (Tables 3,4 , and 5). The stronger relationship between CRP and buccal cancer in our series may be due the high incidence of AQ consumption in our population, making the buccal mucosa the site of greatest risk of contracting malignancy in betel quid chewers $[42,43]$. The intimate contact between the buccal mucosa and the AQ during chewing induces chronic and abnormal mucosa inflammation by promoting the release of inflammatory mediators like IL- 6 , TNF- $\alpha$, and $\mathrm{PGE}_{2}$ by oral keratinocytes [44], playing a crucial role in the pathogenesis of oral cancer.

We believe that there are limitations in this study. The major one comes from the case number in each tumor subsite. However, from this preliminary analysis, we believe that determining CRP levels preoperatively, especially in buccal cancer, would be relevant and useful to clinicians because CRP measurement is rapid, inexpensive, and repeatable in a clinical setting. Using CRP as a biomarker could help clinicians select proper treatment strategies for patients with OSCC, detecting which patients would benefit from adjuvant treatment by predicting pathologically aggressive tumors based on their CRP levels [6].

\section{Conclusions}

The presence of an elevated serum CRP level preoperatively $(\geq 5.0 \mathrm{mg} / \mathrm{L})$ is an important prognostic indicator in oral cancer in the Taiwanese population. Elevated CRP levels are associated with tumor stage and locoregional invasiveness. Furthermore, the prognostic prediction is more evident in buccal cancer, which could be attributed to the tumor's behaviors related with AQ and tobacco use.

\section{Additional files}

Additional file 1: Clinical information of 343 OSCC patients. (SAV 100 kb)

\begin{abstract}
Abbreviations
Cl: Confidence interval; CRP: C-reactive protein; DFS: Disease-free survival; ECS: Extracapsular spread; HR: Hazard ratio; NLR: Neutrophil to lymphocyte ratio; OS: Overall survival; OSCC: Oral cavity squamous cell carcinoma; PET: Positron emission tomography; SCC-Ag: Squamous cell carcinoma antigen; SD: Standard deviation; VEGF: Vascular endothelial growth factor
\end{abstract}

\footnotetext{
Acknowledgements

The authors thank all the members of the Cancer Center and the Tissue Bank at Chang Gung Memorial Hospital, Linkou, for their invaluable assistance.
}

\section{Funding}

This study was supported by grants CMRPG3F0671, CMRPG3F2221, and CMRPB53 from Chang Gung Memorial Hospital and grants NSC 102-2314-B182A-081 and MOST 103-2314-B-182A-057-MY2 from the National Science Council, Executive Yuan, Taiwan, Republic of China. 


\section{Availability of data and materials}

All data generated or analyzed during this study are included in this published article and its supplementary information files.

\section{Authors' contributions}

SFT, HTC, CKY, and SFH conceived the idea for the manuscript, conducted a literature search, and drafted the manuscript. SFH organized the manuscript and critically revised the manuscript. SFT, CKT, AP, KHF, CTL, HMW, CJK, and JTCC collected the data. HTC, CKY, and SFH analyzed the data. HTC plotted the figures. CKT, AP, HMW, CKT, and JTCC critically revised the manuscript. All authors read and approved the final manuscript.

\section{Competing interests}

The authors declare that they have no competing interests.

\section{Consent for publication}

Written informed consent for the publication was obtained from the participants for the images relating to participants in Fig. 1a, b.

\section{Ethics approval and consent to participate}

All patients signed informed consent for participation of this study. This study had ethics approval and consent by the ethic committee in Chang Gung Memorial Hospital (Institutional Review Board of Chang Gung Medical Foundation, IRB No. 201600949B0), Taiwan, Republic of China.

\section{Author details}

'Department of Otolaryngology, Chang Gung Memorial Hospital, Linkou, Taiwan. ${ }^{2}$ Chang Gung University, Taoyuan, Taiwan, Republic of China. ${ }^{3}$ Department of Public Health, Chang Gung University, Taoyuan, Taiwan, Republic of China. ${ }^{4}$ Department of Otolaryngology, Chang Gung Memorial Hospital, Keelung, Taiwan, Republic of China. ${ }^{5}$ Plastic and Reconstructive Surgery, Chang Gung Memorial Hospital, Linkou, Taiwan, Republic of China. ${ }^{6}$ Department of Radiation Oncology, Chang Gung Memorial Hospital, Chang Gung University, Taoyuan, Taiwan, Republic of China. 'Department of Medical Oncology, Chang Gung Memorial Hospital, Chang Gung University, Taoyuan, Taiwan, Republic of China.

Received: 16 November 2016 Accepted: 1 February 2017

Published online: 16 February 2017

\section{References}

1. Bjorkqvist AM, Husgafvel-Pursiainen $\mathrm{K}$, Anttila S, Karjalainen A, Tammilehto L, Mattson $\mathrm{K}$, Vainio $\mathrm{H}$, Knuutila S. DNA gains in $3 q$ occur frequently in squamous cell carcinoma of the lung, but not in adenocarcinoma. Genes Chromosomes Cancer. 1998;22:79-82.

2. Wen CP, Tsai SP, Cheng TY, Chen CJ, Levy DT, Yang HJ, Eriksen MP. Uncovering the relation between betel quid chewing and cigarette smoking in Taiwan. Tob Control. 2005;14:16-22.

3. Bran B, Bran G, Hormann K, Riedel F. The platelet-derived growth factor receptor as a target for vascular endothelial growth factor-mediated antiangiogenetic therapy in head and neck cancer. Int J Oncol. 2009;34:255-61.

4. Il'yasova D, Colbert LH, Harris TB, Newman AB, Bauer DC, Satterfield S, Kritchevsky SB. Circulating levels of inflammatory markers and cancer risk in the health aging and body composition cohort. Cancer Epidemiol Biomarkers Prev. 2005;14:2413-8.

5. Jablonska E, Piotrowski L, Grabowska Z. Serum levels of IL-1b, IL-6, TNFa, sTNF-RI and CRP in patients with oral cavity cancer. Pathol Oncol Res. 1997:3:126-9.

6. Chen HH, Chen IH, Liao CT, Wei FC, Lee LY, Huang SF. Preoperative circulating C-reactive protein levels predict pathological aggressiveness in oral squamous cell carcinoma: a retrospective clinical study. Clin Otolaryngol. 2011;36:147-53.

7. Huang SF, Wei FC, Liao CT, Wang HM, Lin CY, Lo S, Huang JJ, Chen IH, Kang $\mathrm{CJ}$, Chien HT, Chen HH. Risk stratification in oral cavity squamous cell carcinoma by preoperative CRP and SCC antigen levels. Ann Surg Oncol. 2012;19:3856-64.

8. Pepys MB, Hirschfield GM. C-reactive protein: a critical update. J Clin Invest. 2003;111:1805-12.

9. Shrotriya S, Walsh D, Bennani-Baiti N, Thomas S, Lorton C. C-reactive protein is an important biomarker for prognosis tumor recurrence and treatment response in adult solid tumors: a systematic review. PLoS One. 2015;10:e0143080.

10. Coventry BJ, Ashdown ML, Quinn MA, Markovic SN, Yatomi-Clarke SL, Robinson AP. CRP identifies homeostatic immune oscillations in cancer patients: a potential treatment targeting tool? J Transl Med. 2009;7:102.

11. Van Hemelrijck M, Holmberg L, Garmo H, Hammar N, Walldius G, Binda E, Lambe $\mathrm{M}$, Jungner I. Association between levels of C-reactive protein and leukocytes and cancer: three repeated measurements in the Swedish AMORIS study. Cancer Epidemiol Biomarkers Prev. 2011;20:428-37.

12. Edge S, Byrd DR, Compton CC, Fritz AG, Greene FL, Trotti A. AJCC cancer staging manual. American Cancer Society. New York: Springer; 2010.

13. Hsu YP, Hsieh CH, Chien HT, Lai CH, Tsao CK, Liao CT, Kang CJ, Wang HM, Chang JT, Huang SF. Serum markers of CYFRA 21-1 and C-reactive proteins in oral squamous cell carcinoma. World J Surg Oncol. 2015;13:253.

14. Goldstein BY, Chang SC, Hashibe M, La Vecchia C, Zhang ZF. Alcohol consumption and cancers of the oral cavity and pharynx from 1988 to 2009: an update. Eur J Cancer Prev. 2010;19:431-65.

15. Radoi L, Menvielle G, Cyr D, Lapotre-Ledoux B, Stucker I, Luce D, Group IS. Population attributable risks of oral cavity cancer to behavioral and medical risk factors in France: results of a large population-based case-control study, the ICARE study. BMC Cancer. 2015;15:827.

16. Sharan RN, Mehrotra R, Choudhury Y, Asotra K. Association of betel nut with carcinogenesis: revisit with a clinical perspective. PLoS One. 2012;7:e42759.

17. Hashibe M, Brennan P, Chuang SC, Boccia S, Castellsague X, Chen C, Curado MP, Dal Maso L, Daudt AW, Fabianova E, Fernandez L, Wunsch-Filho V, Franceschi S, Hayes RB, Herrero R, Kelsey K, Koifman S, La Vecchia C, Lazarus P, Levi F, Lence JJ, Mates D, Matos E, Menezes A, McClean MD, Muscat J, Eluf-Neto J, Olshan AF, Purdue M, Rudnai P, Schwartz SM, Smith E, Sturgis EM, Szeszenia-Dabrowska N, Talamini R, Wei Q, Winn DM, Shangina O, Pilarska A, Zhang ZF, Ferro G, Berthiller J, Boffetta P. Interaction between tobacco and alcohol use and the risk of head and neck cancer: pooled analysis in the International Head and Neck Cancer Epidemiology Consortium. Cancer Epidemiol Biomarkers Prev. 2009;18:541-50.

18. Scully C, Bedi R. Ethnicity and oral cancer. Lancet Oncol. 2000;1:37-42.

19. Ko YC, Chiang TA, Chang SJ, Hsieh SF. Prevalence of betel quid chewing habit in Taiwan and related sociodemographic factors. J Oral Pathol Med. 1992;21:261-4.

20. Lee S, Choe JW, Kim HK, Sung J. High-sensitivity C-reactive protein and cancer. J Epidemiol. 2011;21:161-8.

21. Ehrsson YT, Hellstrom PM, Brismar K, Sharp L, Langius-Eklof A, Laurell G. Explorative study on the predictive value of systematic inflammatory and metabolic markers on weight loss in head and neck cancer patients undergoing radiotherapy. Support Care Cancer. 2010;18:1385-91.

22. Asegaonkar SB, Asegaonkar BN, Takalkar UV, Advani S, Thorat AP. C-reactive protein and breast cancer: new insights from old molecule. Int J Breast Cancer. 2015;2015:145647.

23. Steffens S, Al Ghazal A, Steinestel J, Lehmann R, Wegener G, Schnoeller TJ, Cronauer MV, Jentzmik F, Schrader M, Kuczyk MA, Schrader AJ. High CRP values predict poor survival in patients with penile cancer. BMC Cancer. 2013;13:223.

24. Steffens S, Kohler A, Rudolph R, Eggers H, Seidel C, Janssen M, Wegener G, Schrader M, Kuczyk MA, Schrader AJ. Validation of CRP as prognostic marker for renal cell carcinoma in a large series of patients. BMC Cancer. 2012;12:399.

25. Pine SR, Mechanic LE, Enewold L, Chaturvedi AK, Katki HA, Zheng YL, Bowman ED, Engels EA, Caporaso NE, Harris CC. Increased levels of circulating interleukin 6, interleukin 8, C-reactive protein, and risk of lung cancer. J Natl Cancer Inst. 2011;103:1112-22.

26. Chaturvedi AK, Caporaso NE, Katki HA, Wong HL, Chatterjee N, Pine SR, Chanock SJ, Goedert JJ, Engels EA. C-reactive protein and risk of lung cancer. J Clin Oncol. 2010;28:2719-26.

27. Fang HY, Huang XY, Chien HT, Chang JT, Liao CT, Huang JJ, Wei FC, Wang HM, Chen IH, Kang CJ, Huang SF. Refining the role of preoperative Creactive protein by neutrophil/lymphocyte ratio in oral cavity squamous cell carcinoma. Laryngoscope. 2013;123:2690-9.

28. Adel M, Tsao CK, Wei FC, Chien HT, Lai CH, Liao CT, Wang HM, Fan KH, Kang CJ, Chang JT, Huang SF. Preoperative SCC antigen, CRP serum levels, and lymph node density in oral squamous cell carcinoma. Medicine (Baltimore). 2016;95:e3149.

29. Lin WH, Chen IH, Wei FC, Huang JJ, Kang CJ, Hsieh LL, Wang HM, Huang SF. Clinical significance of preoperative squamous cell carcinoma antigen in oral-cavity squamous cell carcinoma. Laryngoscope. 2011;121:971-7. 
30. Shamamian P, Schwartz JD, Pocock BJ, Monea S, Whiting D, Marcus SG, Mignatti P. Activation of progelatinase A (MMP-2) by neutrophil elastase, cathepsin $\mathrm{G}$, and proteinase-3: a role for inflammatory cells in tumor invasion and angiogenesis. J Cell Physiol. 2001;189:197-206.

31. Di Carlo E, Forni G, Musiani P. Neutrophils in the antitumoral immune response. Chem Immunol Allergy. 2003;83:182-203.

32. Colotta F, Allavena P, Sica A, Garlanda C, Mantovani A. Cancer-related inflammation, the seventh hallmark of cancer: links to genetic instability. Carcinogenesis. 2009;30:1073-81.

33. Scapini P, Nesi L, Morini M, Tanghetti E, Belleri M, Noonan D, Presta M, Albini A, Cassatella MA. Generation of biologically active angiostatin kringle 1-3 by activated human neutrophils. J Immunol. 2002;168:5798-804.

34. De Larco JE, Wuertz BR, Furcht LT. The potential role of neutrophils in promoting the metastatic phenotype of tumors releasing interleukin-8. Clin Cancer Res. 2004:10:4895-900.

35. Jablonska E, Puzewska W, Grabowska Z, Jablonski J, Talarek L. VEGF, IL-18 and NO production by neutrophils and their serum levels in patients with oral cavity cancer. Cytokine. 2005;30:93-9.

36. Khandavilli SD, Ceallaigh PÓ, Lloyd CJ, Whitaker R. Serum C-reactive protein as a prognostic indicator in patients with oral squamous cell carcinoma. Oral Oncol. 2009;45:912-4.

37. Kruse AL, Luebbers HT, Gratz KW. C-reactive protein levels: a prognostic marker for patients with head and neck cancer? Head Neck Oncol. 2010;2:21.

38. Peter F, Wittekindt C, Finkensieper M, Kiehntopf M, Guntinas-Lichius O Prognostic impact of pretherapeutic laboratory values in head and neck cancer patients. J Cancer Res Clin Oncol. 2013;139:171-8.

39. Chen IH, Liao CT, Wang HM, Huang JJ, Kang CJ, Huang SF. Using SCC antigen and CRP levels as prognostic biomarkers in recurrent oral cavity squamous cell carcinoma. PLoS One. 2014;9:e103265.

40. Farhan-Alanie OM, McMahon J, McMillan DC. Systemic inflammatory response and survival in patients undergoing curative resection of oral squamous cell carcinoma. Br J Oral Maxillofac Surg. 2015;53:126-31.

41. Luo Y, Fu SJ, She DL, Xiong HU, Yang LI. Preoperative C-reactive protein as a prognostic predictor for upper tract urothelial carcinoma: a systematic review and meta-analysis. Mol Clin Oncol. 2015;3:924-8.

42. Lee JJ, Kok SH, Kuo YS, Yang PJ, Hahn LJ. Carcinoma of buccal mucosa-a representative betel nut oral cancer. Formosan J Med. 1997;1:638-47.

43. Chen YK, Huang HC, Lin LM, Lin CC. Primary oral squamous cell carcinoma: an analysis of 703 cases in southern Taiwan. Oral Oncol. 1999:35:173-9.

44. Jeng JH, Wang YJ, Chiang BL, Lee PH, Chan CP, Ho YS, Wang TM, Lee JJ, Hahn LJ, Chang MC. Roles of keratinocyte inflammation in oral cancer: regulating the prostaglandin E2, interleukin- 6 and TNF-alpha production of oral epithelial cells by areca nut extract and arecoline. Carcinogenesis. 2003:24:1301-15.

45. Grimm M, Lazariotou M: Clinical relevance of a new pre-treatment laboratory prognostic index in patients with oral squamous cell carcinoma. Med Oncol 2012, 29: 1435-1447.

\section{Submit your next manuscript to BioMed Central and we will help you at every step:}

- We accept pre-submission inquiries

- Our selector tool helps you to find the most relevant journal

- We provide round the clock customer support

- Convenient online submission

- Thorough peer review

- Inclusion in PubMed and all major indexing services

- Maximum visibility for your research

Submit your manuscript at www.biomedcentral.com/submit

Biomed Central 